\title{
RELAPSING POLYCHONDRITIS WITH EXUBERANT SKIN MANIFESTATIONS - CASE REPORT
}

Millena Almeida Souza Ramos ${ }^{1, \star}$, André Busato da Costa ${ }^{1}$, Kátia Sheylla Malta Purim ${ }^{1}$, Bruna Burko Rocha Chu ${ }^{1}$

1.Universidade Positivo, Curitiba (PR), Brazil.

*Corresponding author: millenaalmeida33@gmail.com

\section{BACKGROUND}

Relapsing polychondritis (RP) is a systemic disease that causes inflammation of cartilage structures and other tissues. The estimated incidence is 3.5 per million, with a peak age between 40 and 50 years. There are few studies addressing cutaneous manifestations of the disease.

\section{CASE REPORT}

Female patient, 42 years old, complained of pain, erythema and edema on right ear for four months (Figure 1). Two months later, she had similar symptoms on the left ear (Figure2). She noted recurrent flares of pruritic erythematous plaques on her back, (Figure 3), chest and neck (Figure 4) that did not respond to antihistamines. Three months after the first symptom, she complained of dyspnea and hoarseness, shoulder and ankle arthralgias and tinnitus. The patient was admitted to the hospital for investigation. Tests results showed C-reactive protein, erythrocyte sedimentation rate and normal audiometry. Antibodies like rheumatoid factor, antinuclear antibody and antineutrophil cytoplasmic antibodies were negative. There were no signs of stenosis on the tracheal tomography. A clinical diagnose of RP was made, based on Damiani and Levine criteria. Biopsy of the skin showed spongiotic dermatitis with a discrete perivascular lymphocytic infiltration, with histiocytes and lymphocytic exocytosis on the dermis. The patient was treated with steroids and had a good response. She was discharged with prednisone $40 \mathrm{mg} /$ day and methotrexate $15 \mathrm{mg} / \mathrm{week}$.

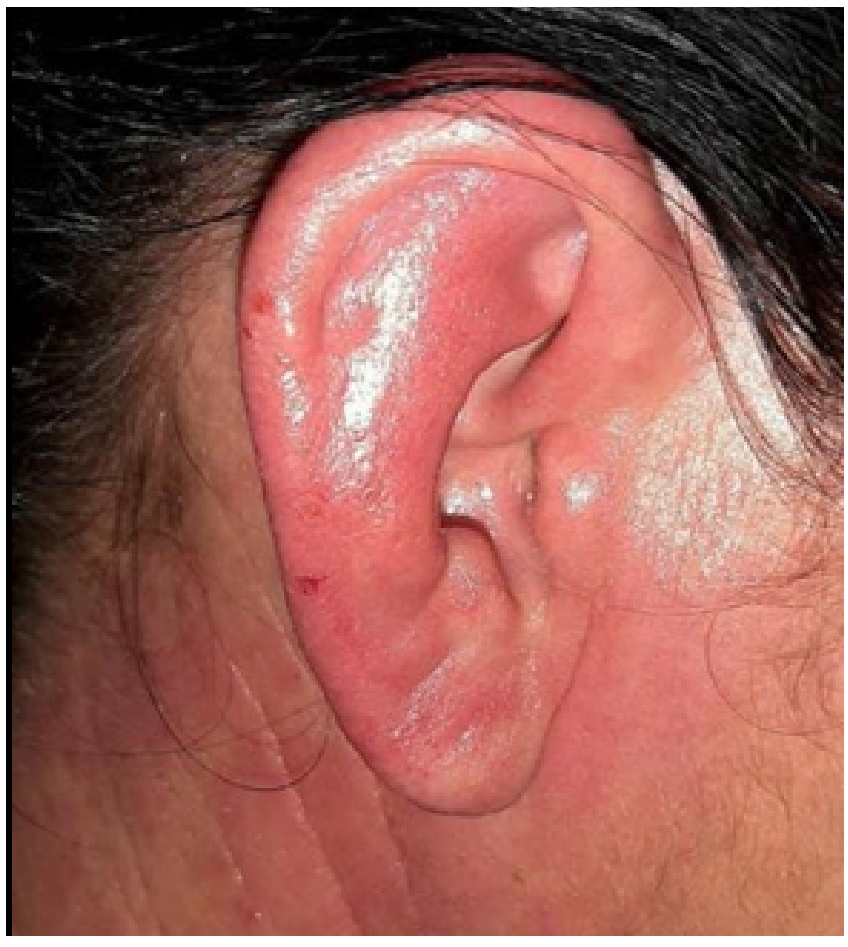

Figure 1. Right ear with signs of inflammation.

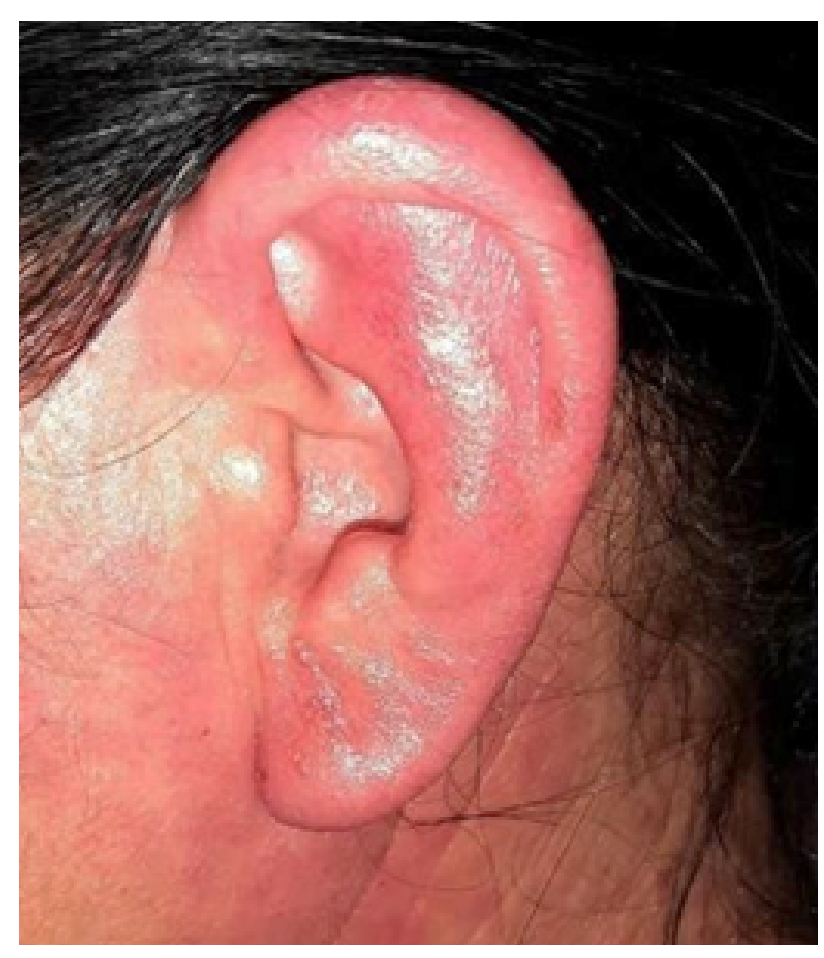

Figure 2. Left ear with signs of inflammation.

Realização: 


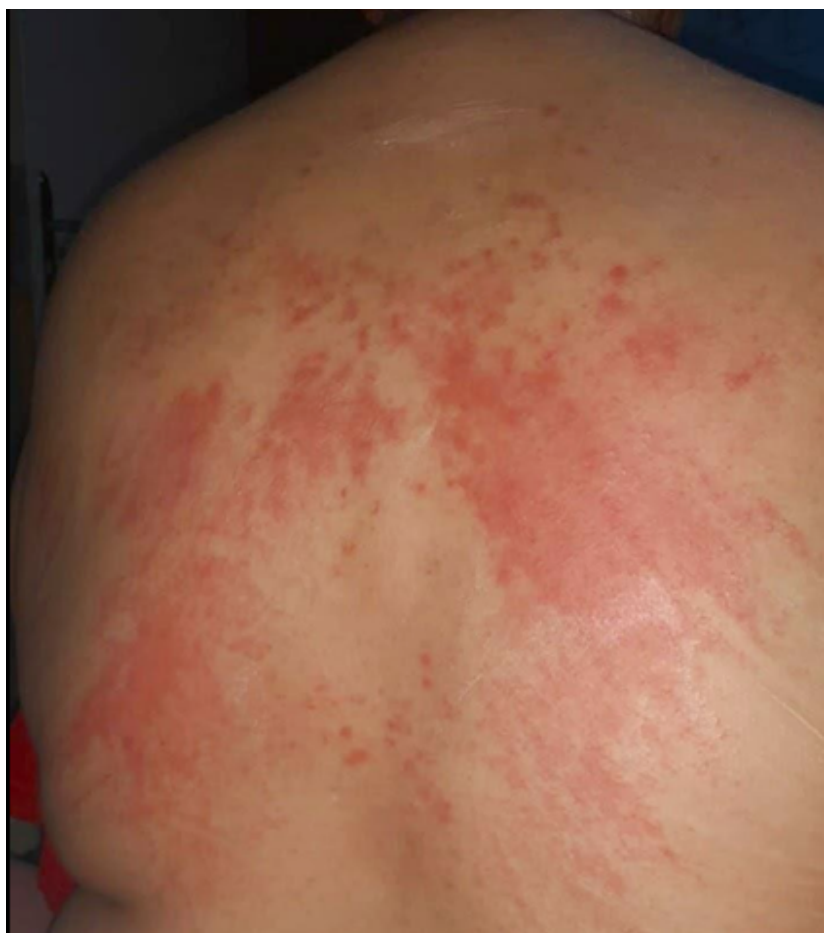

Figure 3. pruritic erythematous plaques on the back.

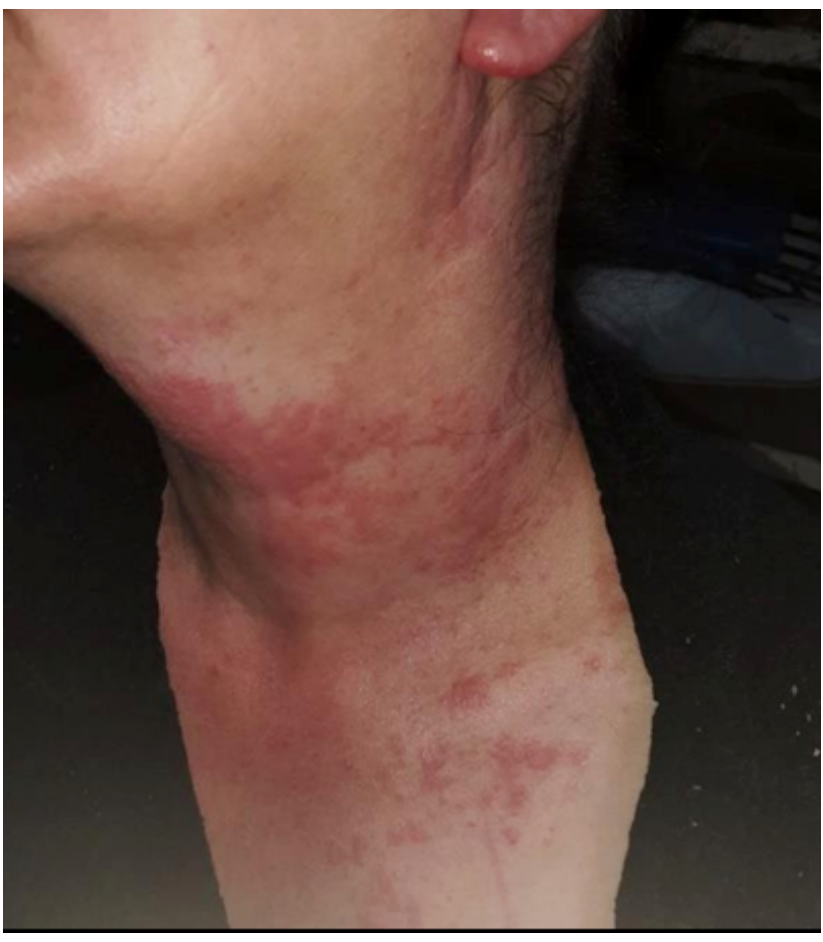

Figure 4. Pruritic erythematous plaques on the neck.

\section{CONCLUSION}

Symptoms of RP include bilateral auricular chondritis, vestibular or auditory dysfunction, nonerosive asymmetric polyarthritis, ocular inflammation, and airway involvement. Dermatological lesions occur in $37 \%$ of cases, such as nodules, purpura, papules, livedo reticularis and distal ulceration, rarely annular urticarial plaques, the latter being related to myelodysplastic syndrome. Skin biopsies may show patterns of leukocytoclastic vasculitis but are often nonspecific. Empirical treatment of moderate RP includes prednisone 1-2 mg/kg/day and immunosuppressants such as methotrexate to spare corticosteroids. This case highlights the importance of considering RP as a possible diagnosis in cases of skin lesions accompanied by signs of ear inflammation.

\section{KEYWORDS}

Relapsing polychondritis, Skin, Lesions. 